Documentation et bibliothèques

DOCUMENTATION BIBLIOTHËQUES

\title{
Les services documentaires dans les sièges sociaux à Montréal
}

\section{Hélène Houde}

Volume 27, numéro 4, décembre 1981

URI : https://id.erudit.org/iderudit/1053796ar

DOI : https://doi.org/10.7202/1053796ar

Aller au sommaire du numéro

Éditeur(s)

Association pour l'avancement des sciences et des techniques de la documentation (ASTED)

\section{ISSN}

0315-2340 (imprimé)

2291-8949 (numérique)

Découvrir la revue

Citer cet article

Houde, H. (1981). Les services documentaires dans les sièges sociaux à

Montréal. Documentation et bibliothèques, 27(4), 149-152.

https://doi.org/10.7202/1053796ar

Tous droits réservés (C) Association pour l'avancement des sciences et des techniques de la documentation (ASTED), 1981
Ce document est protégé par la loi sur le droit d'auteur. L'utilisation des services d'Érudit (y compris la reproduction) est assujettie à sa politique d'utilisation que vous pouvez consulter en ligne.

https://apropos.erudit.org/fr/usagers/politique-dutilisation/ 


\section{chroniques}

\section{Les services documentaires dans les sièges sociaux à Montréal ${ }^{\star}$}

Le marché du travail a longtemps demeuré, pour les bibliothécaires professionnels, la bibliothèque de maison d'enseignement (primaire, secondaire, collégial et universitaire) et la bibliothèque publique. II ne s'est que récemment élargi avec l'apparition, au sein d'organismes divers, des centres de documentation et des bibliothèques.

A l'intérieur de ce nouveau marché, il nous a paru intéressant d'étudier les services documentaires des sièges sociaux:

Le siège social représente le centre de décision d'une entreprise. (...) II s'agit de l'endroit où sont concentrées les activités de direction concourant à la prise de décisions pour l'ensemble de l'entreprise. (...) Le siège social coordonne les activités des unités d'exploitation. (...) La présence de la haute administration est une caractéristique essentielle. (...) En tant que sources d'information à la direction, les services d'études générales (...) se trouvent au siège social ${ }^{1}$.

Si le siège social d'une entreprise est un lieu de décision, il serait normal d'y trouver un centre de documentation ou une bibliothèque pouvant fournir une information de nature à éclairer les prises de décisions. Qu'en est-il exactement? S'il existe dans les sièges sociaux des collections documentaires, quelles en sont les structures et quelles sont les personnes qui en sont responsables?

\section{Cadre d'analyse et méthodologie}

Pour connaître avec précision quels sont les sièges sociaux ayant "pignon sur rue " à Montréal,

\footnotetext{
* Enquête menée dans le cadre d'un cours de maîtrise en bibliothéconomie à l'Université de Montréal et supervisée par M. Marcel Lajeunesse.
}

1. Office de planification et de développement du Québec, Les sièges sociaux et l'emploi au Québec: quelques statistiques partielles, Québec, 1978, p. 7 et 8, passim. nous avons consulté le Financial Post ${ }^{2}$. Parmi les différents tableaux compilés par ses spécialistes, on peut trouver, pour le Canada, la liste des 500 plus grandes entreprises, des 15 plus importants promoteurs, des 25 compagnies d'assurances les plus considérables et des 50 plus grosses banques et institutions financières. Les auteurs classent ces organisations selon l'ordre d'importance du chiffre des ventes ou du revenu des opérations. Nous avons retenu pour les fins de notre enquête toutes celles qui ont un siège social à Montréal et dans la périphérie (Brossard, Lachine, Pointe-Claire, Sorel).

La liste des entreprises consultées apparaît au tableau 1. Elle comprend les entreprises industrielles (1 à 77), le secteur immobilier (11), les compagnies d'assurances (A1-A4), les banques et les institutions financières (B1-B11). Nous avions 93 organismes à contacter; un seul n'a pu être rejoint (Fednav). Notre liste en compte donc 92.

L'enquête a été menée par téléphone à l'automne 1980. Aucune vérification sur place n'a été effectuée : nous avons prêté foi aux renseignements ainsi obtenus. Dans le cadre de cette étude, nous avons adopté la catégorisation du Financial Post 500 sans discuter sa définition du "siège social» («head office»).

L'interview comportait les questions suivantes: 1) $Y$ a-t-il une bibliothèque ou un centre de documentation et qui en est responsable? Un bibliothécaire (lieu d'obtention du diplôme), un bibliotechnicien ou une autre personne ? 2) De quel service relève la bibliothèque ou le centre de documentation? 3) A quel domaine d'activité appartiennent les entreprises qui offrent un service documentaire ? 4) Quelle est la langue de travail ?

Les termes bibliothèque et centre de documen- 
tation n'ont pas été définis a priori. L'enquête a fait ressortir clairement que les interviewés parlaient de bibliothèque lorsque la collection était importante et de centre de documentation dans le cas contraire. Nous avons adopté ces définitions. Les termes "service traditionnel " et "service informatisé » dénotent l'absence ou l'existence de l'automatisation au niveau du prêt et de la référence.

\section{Analyse des résultats}

\section{Nombre de bibliothèques \\ et de centres de documentation}

Des 92 entreprises retenues, 47 ont une bibliothèque ou un centre de documentation. II nous faut soustraire de ces dernières 8 entreprises dont le centre de documentation ou la bibliothèque se trouve hors de Montréal (principalement en Ontario), et une dont le service de documentation est embryonnaire. Donc, 38 sièges sociaux montréalais offrent un service de documentation: 26 d'entre eux ont une bibliothèque et 12 un centre de documentation (tableau 2).

\section{Niveau de formation du personnel et milieu de travail}

Les 26 bibliothèques comptent 21 bibliothécaires professionnels, un bibliotechnicien et 4 personnes diversement qualifiées (secrétaire, commis, etc. ). Le nombre de bibliothécaires diplômés de
l'Université McGill est légèrement plus élevé que celui des diplômés de l'Université de Montréal (13 contre 9). On retrouve aussi des diplômés d'autres universités (Ottawa, Toronto, etc.)

Aucun des 12 centres de documentation n'emploie un bibliothécaire professionnel ou un technicien en documentation. II ne nous a pas été possible de relever la liste des diverses catégories d'emploi affectées aux postes correspondants.

Dans les bibliothèques et les centres de documentation, le milieu de travail est surtout bilingue ou unilingue anglais. Les milieux francophones sont au nombre de 9 (Hydro-Québec, Téléglobe Canada, Banque nationale du Canada, Les Prévoyants, Consolidated Bathurst, Imasco, Sidbec-Dosco, York Lambton, La Sauvegarde).

Les méthodes de travail sont en général traditionnelles, sans automatisation du prêt ou de la référence. Ce sont les bibliothèques des grandes entreprises qui offrent des services informatisés: Canadien Pacifique, Bell Canada, Alcan, Canadien National, Hydro-Québec, Air Canada, Domtar. Tous les centres de documentation fonctionnent selon les méthodes traditionnelles.

\section{Intégration à l'entreprise}

L'enquête téléphonique nous a d'abord révélé que l'unité documentaire, bibliothèque ou centre de documentation, est peu connue dans l'entreprise.

\section{Tableau 1: Liste des entreprises consultées *}

1 Canadien Pacifique
2 Bell Canada
3 Alcan
4 Canadien National
5 Provigo
6 Steinberg
7 Hydro-Québec
8 Seagram
9 Air Canada
10 Domtar
11 Genstar
12 Consolidated-Bathurst
13 Molson
14 CIP
15 CIL
16 Du Pont du Canada
17 Anglo-Can. Telephone
18 Imasco
19 Petrofina
20 Dominion Textile
21 Ciment Lafarge
22 Société générale
de financement
23 Métro-Richelieu
24 Kraft
25 Sidbec
26 Canron

1 Canadien Pacifique

2 Bell Canada

4 Canadien National

5 Provigo

6 Steinberg

7 Hydro-Québec

8 Seagram

9 Air Canada

13 Molson

14 CIP

17 Anglo-Can. Telephone

18 Imasco

19 Petrofina

23 Métro-Richelieu

26 Canron
27 Pratt \& Whitney

28 Bombardier

29 Celanese

30 Johns-Manville

31 Power Corporation

32 Robin Hood

33 Kruger Pulp \& Paper

34 Ciment St-Laurent

35 Allied Chemical

36 Dominion Dairies

37 Fednav

38 Ingersoll-Rand

39 Can. Reynolds Metals

40 Reitman's

41 Drummond McCall

42 Marks \& Spencer

43 BASF

44 Asbestos Corp.

45 York Lambton

46 Crane

47 Quebecor

48 Rolland

49 Allis-Chalmers

50 Henry Birks

51 Ronalds-Federated

52 Brooke Bond Foods

53 UAP
54 Canadian Liquid Air

55 Maislin Industries

56 QIT-Fer et Titane

57 SNC Group

58 Culinar

59 Ciba-Geigy

60 Chromasco

61 Sintra

62 Nordair

63 Canadair

64 Monenco

65 Marconi

66 Resource Service Group

67 Wabasso

68 Peerless Rug

69 Canada Starch

70 Benson \& Hedges

71 Teleglobe Canada

72 Hoechst Canada

73 Boveri

74 Johnson \& Johnson

75 Canadian Salt

76 Cassidy's Ltd.

77 International Paints
11 Trizec Corp.

A1 Standard Life

A2 La Prudentielle

A3 Alliance Mutual Life

A4 La Sauvegarde

B1 Banque Royale

B2 Banque de Montréal

B3 Banque Nationale du Canada

B4 Montreal City and District Savings Bank

B5 Banque Mercantile

B6 Federal Business Development Bank

B7 Montreal Trust

B8 Roymor Mortgage Corp.

B9 Trust Général du Canada

B10 Roynat

B11 Prenor Group (Les Prévoyants) 
Nous devions souvent converser assez longuement avec la téléphoniste pour être bien dirigée dans nos recherches. En plusieurs occasions, nous avions un premier contact avec le service de relations publiques.

Les fonctions des bibliothèques et des centres de documentation sont en outre mal définies. Cette impression se confirme par le nombre d'unités administratives auxquelles ils peuvent être rattachés: au total 15 unités différentes.

L'unité la plus souvent citée est le service de la recherche: cette affiliation nous semble la plus adéquate. Les services auxiliaires, qui regroupent habituellement les services de soutien de l'entreprise (secrétariat, informatique...) partagent le deuxième rang avec les cas de «décentralisation", qui correspond à la situation des entreprises dotées de plusieurs petits centres de documentation indépendants les uns des autres et ne desservant que l'unité dont ils relèvent. Fait à noter, trois personnes n'ont pu nous indiquer de quelle unité administrative dépendait la bibliothèque ou le centre de documentation.

\section{Domaines d'activité}

Remodelant la classification employée par le Bureau de la statistique du Québec ${ }^{3}$, nous avons tenté de cerner les types d'entreprises où il existe un service documentaire (tableau 5). Ce sont principalement les banques et institutions financières (7), les entreprises de produits chimiques (7) et les compagnies d'assurances (4). Pourquoi? Une bonne situation financière, bien que facteur important, ne saurait à elle seule expliquer le phénomène. Tout porte à croire que les exigences de la recherche, l'obligation d'être à la fine pointe des connaissances (par exemple en matière d'expérimentation chimique, de conjoncture éco-

3. Bureau de la statistique du Québec, Statistiques principales des manufacturiers québécois, 1980.

\section{Tableau 2 : Bibliothèques et centres de documentation dans les sièges sociaux à Montréal}

RANG NOM DE L'ENTREPRISE BP T A UNIV TR IF L SERVICE RESPONSABLE DOMAINE D'ACTIVITE

\section{BIBLIOTHËQUES}

1 Canadien Pacifique

Bell Canada

Alcan

Canadien National

Hydro-Québec

Air Canada

Domtar

CIL

Sidbec

Pratt \& Whitney

Celanese

Canadian Liquid Air

QIT - Fer et Titane

SNC Group

Ciba-Geigy

Canadair

Monenco

Marconi

Teleglobe Canada

Hoechst

La Sauvegarde

Banque Royale

Banque de Montréal

Banque Nationale du Can.

Federal Business Develop.

Prenor (Prévoyants)

\section{CENTRES DE DOCUMENTATION}

$\begin{array}{ll}12 & \text { Consolidated-Bathurst } \\ 14 & \text { Cie Internat. de papier } \\ 18 & \text { Imasco } \\ 31 & \text { Power Corporation } \\ 43 & \text { BASF } \\ 45 & \text { York Lambton } \\ 74 & \text { Johnson \& Johnson } \\ \text { A1 } & \text { Standard Life } \\ \text { A2 } & \text { La Prudentielle } \\ \text { A3 } & \text { Alliance } \\ \text { B5 } & \text { Mercantile } \\ \text { B7 } & \text { Montreal Trust }\end{array}$
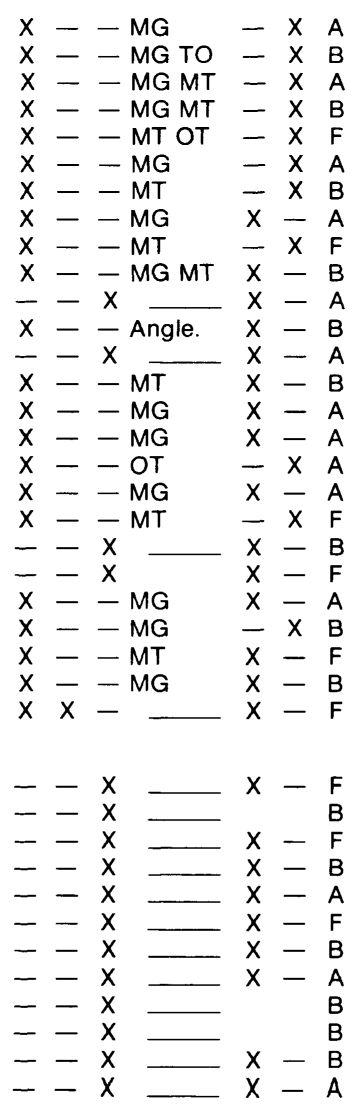

Relations publiques

Le Secrétariat

V.-p. à l'administration

Service de la gestion

Le Secrétariat

Services auxiliaires

La Recherche

Planification

Recherche et développ.

Génie

Services auxiliaires

La Recherche

Services auxiliaires

Medical Service

La Recherche

V. $-p$. à l'administration

Services auxiliaires

Services auxiliaires

Communications

Économie et développ.

Services auxiliaires

Communications

La Recherche

Communications

Traduction

Décentralisation

Décentralisation

La Recherche

V.-p. à l'administration

Contentieux

La Recherche

Décentralisation

Décentralisation

Décentralisation

Crédit
18. Transport

16. Service public

08. Produits métalliques

18. Transport

16. Service public

18. Transport

15. Construction-ingén.

14. Produits chimiques

07. Transf. des métaux

10. Equipement-transp.

14. Produits chimiques

14. Produits chimiques

07. Transf. des métaux

15. Construction-ingén.

14. Produits chimiques

10. Equipement-transp.

15. Construction-Ingén.

11. Produits chimiques

16. Service public

14. Produits chimiques

21. Assurances

22. Banques et IF

22. Banques et IF

22. Banques et IF

22. Banques et IF

22. Banques et IF

05. Papier

05. Papier

01. Aliments et boissons

17. Industries diverses

14. Produits chimiques

17. Industries diverses

14. Produits chimiques

21. Assurances

21. Assurances

21. Assurances

22. Banques et IF

22. Banques et IF 


\begin{tabular}{|lrc|}
\hline \multicolumn{3}{|c|}{ Tableau 3 } \\
Niveau de formation du personnel \\
et milieu de travail \\
\hline Bibliothèque & $\begin{array}{c}\text { Centre de } \\
\text { documentation }\end{array}$ \\
Bibliothécaires professionnels & 21 & 0 \\
Bibliotechniciens & 1 & 0 \\
Autres & 4 & 12 \\
Bilinguisme & 9 & 6 \\
Unilinguisme anglais & 11 & 3 \\
Unilinguisme français & 6 & 3 \\
Méthodes traditionnelles & 15 & 12 \\
Méthodes automatisées & 11 & 0 \\
\hline
\end{tabular}

\begin{tabular}{|c|c|c|c|}
\hline $\begin{array}{l}\text { Unités administr } \\
\text { services }\end{array}$ & & $\begin{array}{l}\text { au } 4 \\
\text { s dont relèvent les } \\
\text { umentaires }\end{array}$ & \\
\hline Recherche & 7 & Génie & 1 \\
\hline Services auxiliaires & 5 & Gestion & 1 \\
\hline Communications & 3 & Service médical & 1 \\
\hline Vice-présidence & & & \\
\hline adm. & 3 & Planification & 1 \\
\hline Secrétariat & 2 & Relations publiques & 1 \\
\hline Contentieux & 1 & Traduction & 1 \\
\hline Crédit & 1 & & \\
\hline $\begin{array}{l}\text { Direction générale } \\
\text { Économie et }\end{array}$ & 1 & Décentralisation & 5 \\
\hline développement & 1 & Sans réponse & 3 \\
\hline
\end{tabular}

\begin{tabular}{|c|}
\hline Tableau 5 \\
Nombre de centres de documentation \\
ou de bibliothèques par rapport au nombre \\
d'entreprises consultées dans chaque domaine
\end{tabular}

1. Aliments et boissons

$1 / 9$

2. Tabac

$0 / 1$

3. Textile

$0 / 2$

4. Vêtement

$0 / 1$

5. Papier

2/4

6. Imprimerie

$0 / 2$

7. Transformation des métaux

$2 / 2$

8. Produits métalliques

9. Machinerie

10. Equipement-transport

11. Produits électriques

12. Produits minéraux

13. Produits pétroliers

14. Produits chimiques

15. Construction-ingénierie

16. Services publics

17. Industries diverses

18. Transport

19. Commerce-détail

20. Immobilier

21. Assurances

22. Banques et institutions financières nomique) amènent les entreprises à se doter d'un service documentaire.

Les grands «absents " sont les entreprises des secteurs «aliments et boissons» et «commerce de détail ": Provigo, Steinberg, Culinar, Robin Hood, Kraft, Dominion Dairies, etc.

\section{Conclusion}

L'étude démontre que $41,1 \%$ des plus grandes entreprises ayant un siège social à Montréal offrent un service documentaire. Ce pourcentage, sans être élevé, peut paraître satisfaisant. Mais en y regardant de plus près, on constate que seulement 21 des 92 entreprises consultées embauchent des bibliothécaires professionnels, soit $22,8 \%$; la situation s'avère alors beaucoup moins encourageante. Sans être pessimiste, comment espérer, avec les restrictions budgétaires qui sévissent actuellement dans le monde des affaires, une amélioration de la situation? Tout au plus peut-on souhaiter l'intégration de bibliothécaires professionnels dans les centres de documentation déjà existants, puisque les entreprises ayant des centres de documentation confient leur service à des personnes sans formation professionnelle. Par contre, presque toutes les bibliothèques sont confiées à des bibliothécaires professionnels $(21 / 26)$.

Sur la base de l'ensemble des services documentaires (bibliothèques et centres de documentation), on peut conclure surtout que les sièges sociaux montréalais ne sont pas, actuellement, un important marché pour le bibliothécaire professionnel, même si l'on trouve couramment des services documentaires dans les entreprises tournées vers les transports (Canadien Pacifique, Canadien National, Air Canada) et les produits chimiques (CIL, Celanese, BASF, Air Liquide, Ciba, Hoescht, Johnson \& Johnson), ainsi que dans les banques, institutions financières et compagnies d'assurances. D'autre part, les services documentaires sont rattachés à des unités administratives fort différentes. Enfin, le bibliothécaire professionnel travaille dans un milieu bilingue où le service est habituellement traditionnel.

Cette étude se voulait un simple tour d'horizon. Les conclusions que nous en tirons ne doivent pas engendrer un pessimisme exagéré. Le bibliothécaire professionnel ne trouve peut-être pas actuellement d'emploi dans le monde des sièges sociaux montréalais, mais le spécialiste en sciences de l'information saura peut-être un jour s'y faire une place.

\author{
Hélène Houde \\ Centre de documentation \\ INRS-Urbanisation \\ Montréal
}

